\title{
Sucrose acceptance and different forms of associative learning of the honey bee (Apis mellifera L.) in the field and laboratory
}

\author{
Samir Mujagic*, Jana Sarkander, Barbara Erber and Joachim Erber
}

Institut für Ökologie, Technische Universität Berlin, Berlin, Germany

Edited by:

Jean-Marc Devaud, Centre National de la Recherche Scientifique - University

Paul Sabatier, France

\section{Reviewed by:}

Edith Roussel, Université Paul Sabatier, France

Natalie Hempel de Ibarra, The

University of Exeter, UK

*Correspondence:

Samir Mujagic, Department of Biological Cybernetics, Faculty of

Biology, University of Bielefeld, PO Box 1001 31, Bielefeld D-33501, Germany. e-mail:samir.mujagic@uni-bielefeld.de
The experiments analyze different forms of learning and 24-h retention in the field and in the laboratory in bees that accept sucrose with either low $(\leq 3 \%)$ or high $(\geq 30 \%$ or $\geq 50 \%$ ) concentrations. In the field we studied color learning at a food site and at the hive entrance. In the laboratory olfactory conditioning of the proboscis extension response (PER) was examined. In the color learning protocol at a feeder, bees with low sucrose acceptance thresholds ( $\leq 3 \%)$ show significantly faster and better acquisition than bees with high thresholds $(\geq 50 \%)$. Retention after $24 \mathrm{~h}$ is significantly different between the two groups of bees and the choice reactions converge. Bees with low and high acceptance thresholds in the field show no differences in the sucrose sensitivity PER tests in the laboratory. Acceptance thresholds in the field are thus a more sensitive behavioral measure than PER responsiveness in the laboratory. Bees with low acceptance thresholds show significantly better acquisition and 24-h retention in olfactory learning in the laboratory compared to bees with high thresholds. In the learning protocol at the hive entrance bees learn without sucrose reward that a color cue signals an open entrance. In this experiment, bees with high sucrose acceptance thresholds showed significantly better learning and reversal learning than bees with low thresholds. These results demonstrate that sucrose acceptance thresholds affect only those forms of learning in which sucrose serves as the reward. The results also show that foraging behavior in the field is a good predictor for learning behavior in the field and in the laboratory.

Keywords: Apis mellifera, sucrose acceptance, color learning, olfactory PER conditioning, retention, hive entrance learning, reward value

\section{INTRODUCTION}

In their natural environment honey bees rapidly learn the features and locations of profitable floral sources and are able to remember this information for a long period of time (von Frisch, 1965; Waser, 1986). Associative learning of individual bees is a prerequisite for the exploitation of profitable food sources and plays a pivotal role in the organization and division of labor in a bee colony (for a review see Page and Erber, 2002). Nectar is the main food source of honey bee colonies. It also serves as a reward for individual forager bees and reinforces the association of sensory signals with nectar resources (von Frisch, 1965; Menzel and Müller, 1996). Honey bees are able to discriminate the different sugar components in nectar and prefer sucrose to most other naturally occurring sugars (Barker and Lehner, 1974). Therefore, most learning protocols with bees in the field or in the laboratory use sucrose solutions as the reward.

The physiological mechanisms of learning in the bee have been investigated using different learning protocols under laboratory conditions. In the laboratory bees can be conditioned to olfactory, visual and tactile cues. For olfactory conditioning the proboscis extension response (PER) protocol with harnessed bees has been used for over six decades (Frings, 1944; Vareschi, 1971; Bitterman et al., 1983). This protocol is based on the PER which can be elicited

Abbreviations: GRS, gustatory response score; PER, proboscis extension response. by stimulation of the antennae with sucrose. When presentation of an odor is paired with sucrose stimulation of the antennae and proboscis, the bee responds after $2-5$ pairings with the conditioned PER. There exist also different protocols for color conditioning of honey bees in the laboratory which either need a large number of learning trials (Masuhr and Menzel, 1972) or removal of the antennae (Hori et al., 2006) or the flagellae (Niggebrügge et al., 2009). Very effective laboratory conditioning protocols were developed for tactile PER conditioning and for operant conditioning of antennal movements (Erber et al., 1998; Kisch and Erber, 1999; Kisch and Haupt, 2009).

Individual bees can differ significantly in their sensitivities for sucrose which can be estimated under laboratory conditions using the sucrose concentration dependent PER (Page et al., 1998; Scheiner et al., 2003). In these experiments a harnessed bee is stimulated with increasing concentrations of sucrose and it is registered at which concentrations PER occurs (Page et al., 1998). Sensitive bees respond at low sucrose concentrations or even when stimulated with water, while insensitive bees show the PER only for higher concentrations of sucrose. Individual thresholds for sucrose or individual sucrose sensitivity in bees can be estimated using these tests (Page et al., 1998). Sucrose sensitivity is strongly correlated with the foraging task specialization of a bee. Pollen and water foragers have the highest sensitivity for sucrose, while nectar foragers have 
a lower sensitivity (Pankiw and Page, 2000). In the field individual nectar bees accept different sucrose concentrations while foraging at a food site (von Frisch, 1927; Núñez, 1966; Mujagic and Erber, 2009). Very sensitive bees collect low sucrose concentrations and even water, while insensitive bees collect only high sucrose concentrations (Mujagic and Erber, 2009). Acceptance for sucrose in the field and sucrose responsiveness of the same bees in the laboratory show only partial correlation (Mujagic and Erber, 2009). Nectar foragers which differ significantly in their sucrose acceptance in the field can show similar sucrose responsiveness in the laboratory, suggesting that acceptance measured in the field is a more sensitive parameter than the concentration dependence of the PER measured in the laboratory (Mujagic and Erber, 2009).

The individual differences of sucrose responsiveness have consequences for learning behavior. As sucrose is the reward in most conditioning protocols in the field and in the laboratory, the reward value during conditioning is different in bees that have low or high sucrose sensitivity. The relations between sucrose sensitivity and learning were analyzed in a series of laboratory studies using different classical and operant conditioning protocols (Scheiner et al., 1999, 2001a,b, 2003, 2005). The laboratory experiments demonstrated that sucrose PER sensitivity is correlated with learning performance. Bees which were highly responsive to sucrose stimuli applied to the antennae had significantly better acquisition and retention than bees with low sucrose sensitivity. These experiments support the hypothesis that the reward value of sucrose during associative learning depends on the individual sensitivity for sucrose (Scheiner et al., 2005). It is not clear at the moment whether the rules between sucrose sensitivity and associative learning that were found under laboratory conditions are also valid in the field. A correlation between sucrose sensitivity in the field and associative learning at a food site would have significant consequences for the division of foraging labor within a colony.

It is the main goal of the present study to analyze the effects of different sucrose sensitivities on color learning in the field. We want to compare color learning at a food site where sucrose serves as a reward with color learning at the hive entrance which functions without sucrose reward. With these experiments we want to test two alternative hypotheses concerning the relations between sucrose sensitivity and learning. If differences in learning performance were only found with a sucrose reward, this would imply that different reward values cause differences in learning performance and memory formation. If differences in learning performance can be found also when sucrose is not the reward, this would imply that different sensory sensitivities also affect the physiological mechanisms of learning. Color learning at a food site is a good protocol to test the effects of sucrose rewards on learning performance in the field. Free flying bees learn color signals very fast. They reach the asymptote of the acquisition curve after 3-10 rewards and can discriminate the conditioned color very efficiently from an unrewarded alternative (Menzel, 1969; Erber, 1975a). Even very short sucrose rewards lasting only $100 \mathrm{~ms}$ are sufficient for successful association (Erber, 1975a,b). In the hive entrance color learning protocol sucrose does not serve as a reward. The bees learn that a visual cue signals an open entrance to the hive. The return to the colony functions as the reward in this protocol. This paradigm has been successfully used to analyze visual learning, discrimination and route memory in different hymenoptera (Schremmer, 1941; Beier and Menzel, 1972; Chittka and Menzel, 1992; Harris et al., 2005; Zhang et al., 2006).

It is another goal of this study to investigate whether different sucrose sensitivities in the field have consequences for learning performance in the laboratory. Previous experiments have shown that sucrose sensitivity measured with the PER test affects learning performance under laboratory conditions (Scheiner et al., 1999, 2001a,b, 2003, 2005). It is so far not known whether differences of sucrose acceptance in the field have similar effects on learning performance in the laboratory. For learning studies under laboratory conditions we decided to use olfactory PER conditioning because similar to the conditions in the field, bees can be conditioned to a single sensory cue and then tested with two alternative cues. Recently published color conditioning protocols for the laboratory (Hori et al., 2006; Niggebrügge et al., 2009) could not be used for these experiments because the bees have to be kept for at least 1 day in tubes and the antennae or parts of the antennae have to be cut off before conditioning. Under these conditions it is impossible to measure sucrose responsiveness in the laboratory. It is also impossible to correlate sucrose acceptance in the field with behavior in the laboratory that is measured 1 or 2 days after the field tests.

In the last years laboratory experiments on sensory sensitivity in bees were used to develop hypotheses concerning foraging and learning behavior in a natural environment (Scheiner et al., 2004). With the present study we want to examine whether the findings from the laboratory are valid also under field conditions and whether sucrose sensitivity in the field affects learning in free flying (color learning at food site) and harnessed bees (PER odor conditioning). So far, sucrose served as a reward in all experiments analyzing the relations between sucrose sensitivity and learning. With this study we want to examine for the first time whether bees with different sucrose acceptance also show differences in learning performance when sucrose is not the reward (hive entrance color conditioning). These experiments will bridge the gaps between behavioral studies under laboratory and field conditions. They will also test whether conclusions which were developed for learning under laboratory conditions hold in the natural environment.

\section{MATERIALS AND METHODS}

We used three different learning protocols in which bees were either conditioned to a color in the field or to an odor in the laboratory. In all three protocols a single sensory cue is rewarded and discrimination is tested in dual choice tests by offering simultaneously two alternative colors or sequentially two different odors. In a first set of experiments we studied color conditioning at a food site in the field. We measured acquisition, discrimination and 24-h retention in free flying bees which either accepted $\geq 50 \%(\mathrm{w} / \mathrm{w})$ or $\leq 3 \%(\mathrm{w} / \mathrm{w})$ sucrose in the field. In a second set of experiments we selected free flying bees with sucrose acceptance thresholds $\geq 50 \%$ (w/w) or $\leq 3 \%$ $(\mathrm{w} / \mathrm{w})$. The bees were then transferred to the laboratory for experiments using the olfactory PER conditioning protocol. Similar to the learning experiments at the food site, acquisition, discrimination and 24 -h retention were analyzed in these bees. In the third set of experiments we tested free flying bees which had different sucrose acceptance $(\geq 30 \%$ or $\leq 3 \%)$ in a hive entrance color learning protocol which functions without sucrose reward. 


\section{SELECTING BEES WITH DIFFERENT ACCEPTANCE THRESHOLDS}

For the color learning experiments in the field and the olfactory PER conditioning experiments in the laboratory we used bees from a large hive. At the beginning of an experiment acceptance thresholds for sucrose were measured in individual bees. Honey bees from the colony were trained to visit a food site in a green house approximately $50 \mathrm{~m}$ away from the hive. The bees visiting the feeder were marked individually with color-coded spots on the thorax and abdomen. The feeder contained $50 \%(\mathrm{w} / \mathrm{w})$ sucrose solution. To determine the individual acceptance threshold, the concentration of the sucrose solution at the feeder was reduced in steps (for details see also Mujagic and Erber, 2009). All the experiments were performed with newly alarmed nectar foragers, while bees belonging to the long-term foraging group that visited the food site regularly, were caught and kept in a box for the duration of the experiment. The newly alarmed bees were offered each of the following concentrations for $20 \mathrm{~min}: 50 \%, 30 \%, 10 \%$ and $3 \%(\mathrm{w} / \mathrm{w})$. The exact sucrose concentration was adjusted using a refractometer (N-50 E, Atago, Tokyo, Japan). It was recorded at which concentrations an individual bee visited the feeder. After testing each concentration for $20 \mathrm{~min}$, the bees were assigned to one of two groups. Bees which accepted sucrose concentrations of $50 \%(\mathrm{w} / \mathrm{w})$ but no smaller concentrations were assigned to the "high threshold" or "insensitive" group ( $\geq 50 \%$ acceptance group). Bees which accepted a concentration of $3 \%(\mathrm{w} / \mathrm{w})$ were assigned to the "low threshold" or "sensitive" group ( $\leq 3 \%$ acceptance group). Bees which accepted $30 \%(w / w)$ or $10 \%(\mathrm{w} / \mathrm{w})$ but no lower concentrations were not used for the color learning experiments at the food site or the olfactory conditioning experiments. After the tests of acceptance, a single bee was selected if color learning at the food site was analyzed. For the olfactory conditioning experiments in the laboratory several bees with acceptance thresholds of $\geq 50 \%$ or $\leq 3 \%$ were caught at the feeder after drinking for $20 \mathrm{~s}$ either the $50 \%$ or $3 \%$ sucrose solution. The bees were placed in individual glass vials and transported to the laboratory (for details see Experiment 2: Olfactory PER conditioning in the laboratory)

\section{EXPERIMENT 1: COLOR LEARNING AT A FOOD SITE IN THE FIELD}

At random a single, newly alarmed bee which belonged either to the $\geq 50 \%$ or the $\leq 3 \%$ acceptance group was chosen and used for the following experiments. All other bees visiting the feeder were caught and kept in a box during the experiment. Similar to the study of Menzel (1967) we used horizontal color cues for conditioning and testing. The cues were produced by LEDs illuminating semitransparent Plexiglas -plates $(10 \times 10 \mathrm{~cm})$ from below. Homogenous illumination was produced by placing each plate on a $5 \mathrm{~cm}$ high cylinder which had 40 LEDs arranged in two stripes around the inner circumference. The LEDs emitted either green light $(\lambda=525 \mathrm{~nm}$; LM 10A-T2-LINEARlight Flex, 24V-1200 lm-72W, Osram GmbH, Munich, Germany) or blue light ( $\lambda=470 \mathrm{~nm}$; LM 10A-B2-LINEARlight Flex, 24V-460 lm-48W, Osram GmbH, Munich, Germany). Four independent LED illumination units each driven by a constant current source were used. The experiments were performed on a white table $(100 \times 100 \mathrm{~cm})$ inside a shady green house which had a window to allow bees to fly in and out. To avoid olfactory cues due to scent marking of the bees, the plates used for conditioning and testing were cleaned regularly with $70 \%$ ethanol and water. The illumination units used during the conditioning trials were never used during the unrewarded test trials. For conditioning an illumination unit with green light was placed in the center of the table and a small feeder containing 50\% sucrose solution was placed on the plate. The bee could land on the green plate with the feeder and drink the solution ad libitum. For the color choice tests two units, one illuminated with green and the other with blue light, were placed approximately $50 \mathrm{~cm}$ apart from each other on the table. Each of the units had an empty feeder in the center of the plate.

At the beginning of the experiment spontaneous choice preference for the two colors was tested. Two light sources with green and blue illumination were placed on the table and for $1 \mathrm{~min}$ it was registered how often the experimental bee approached each of the two color alternatives. Then the positions of the two color plates were exchanged and behavior was registered again for $1 \mathrm{~min}$. An approach was counted when the bee flew over the illuminated plate or hovered around the feeder in the center. Landings on the plates were counted separately but as bees did not land frequently, this measure was not used for evaluation of the experiments. After measuring spontaneous choice, the bee was conditioned for the first time. Irrespective of the acceptance group to which a bee belonged, it was rewarded with $50 \%$ sucrose in the feeder on a green light source positioned in the middle of the table. The bee flew back to the hive and color choice was tested after it returned to the test site in the same way as described for spontaneous choice. Each bee was conditioned seven times and color choice was tested after each conditioning trial when the bee returned to the set-up. After the color test following the seventh trial, the test bee was caught and put in a small queen cage $(10 \mathrm{~cm} \times 3 \mathrm{~cm} \times 1.5 \mathrm{~cm})$ which was then placed for $24 \mathrm{~h}$ inside the home colony. On the next day the cage was taken from the colony and the bee was released from the cage inside the green house. All tested animals flew back to the hive and usually returned to the green house after 10-30 min. Bees returning to the set-up later than $30 \mathrm{~min}$ after release were discarded from the following retention test. In the $24 \mathrm{~h}$ retention test color choice of the conditioned bee was measured as described before.

\section{EXPERIMENT 2: OLFACTORY PER CONDITIONING IN THE LABORATORY}

Nectar foragers with sucrose acceptance thresholds of $\geq 50 \%$ or $\leq 3 \%$ in the field were selected and tested in an absolute olfactory PER conditioning experiment with additional dual choice test in the laboratory. Similar to color learning with free flying bees in the field, seven conditioning trials and 24-h retention were analyzed. After testing the sucrose acceptance threshold, each bee was caught at the feeder after drinking for $20 \mathrm{~s}$ either the offered $50 \%$ or $3 \%$ sucrose solution. In a previous experiment (Mujagic, 2009) we measured at a feeder in the field the ingested volume/time for sucrose solutions with different concentrations ( $50 \%$ and $3 \%$ ). We found that bees from the two acceptance groups drink approximately the same volumes/time $(\approx 1 \mu \mathrm{l} / \mathrm{s})$. We also determined the crop content and found no significant differences between the crop volumes for both sucrose concentrations.

In the present study bees were allowed to drink for $20 \mathrm{~s}$ which leads to equally filled crops in the tested bees. The exact drinking duration was measured with a stopwatch. All captured bees were kept individually in small glass vials and taken immediately to the 
laboratory. The bees were shortly cooled in a refrigerator at $4{ }^{\circ} \mathrm{C}$ until they showed the first signs of immobility. Then they were placed in small metal holders with strips of adhesive tape attached between head and thorax and over the abdomen. Bees were allowed to recover for at least $15 \mathrm{~min}$ before testing sucrose responsiveness. In this test the antennae of the harnessed bee were stimulated with a droplet of water and increasing sucrose concentrations of $0.1 \%$, $0.3 \%, 1 \%, 3 \%, 10 \%, 30 \%$ and $50 \%(\mathrm{w} / \mathrm{w})$. It was registered at which concentrations PER occurred (for details see Mujagic and Erber, 2009). The temporal interval between stimulations was $2 \mathrm{~min}$. Responsiveness in this test can be used to compare in the same bee sucrose responsiveness in the laboratory with acceptance in the field. The gustatory response score (GRS) is a good measure for responsiveness in the laboratory (Scheiner, 2004). This score is defined as the sum of proboscis extensions elicited by water and the different sucrose concentrations during the sucrose concentration PER test. As eight different gustatory stimuli were applied, the GRS can vary between 0 (a bee not responding with PER to any stimulus) and 8 (a bee responding to all stimuli). Bees that did not respond to any stimulus (GRS 0) were excluded from the experiment.

Olfactory PER conditioning started 15 min after the sucrose concentration PER test. For olfactory PER conditioning we used cineole ( $\geq 98 \%$, C8144-Sigma-Aldrich Chemie GmbH, Munich, Germany) as CS+ and clove oil (C8392-Sigma-Aldrich Chemie $\mathrm{GmbH}$, Munich, Germany) as CS-. The experimental bee was placed in front of two tubes (diameter $5 \mathrm{~mm}$ ) through which a constant airstream from an aquarium pump was blown. An odor was added to the airstream by opening the valve of a channel that contained a piece of cellulose soaked with either $1 \mu \mathrm{l}$ of cineole or $1 \mu \mathrm{l}$ of clove oil. The scented air was removed with an exhaust vent placed behind the bee. Olfactory PER conditioning started with a test of spontaneous PER behavior. Each of the two test odors was presented for $5 \mathrm{~s}$ to the bee at a stimulus interval of $5 \mathrm{~min}$. Bees responding with PER during the spontaneous test were discarded from the following conditioning assay. The bees were then conditioned in seven trials to the CS+ (cineole). In each trial the CS+ was presented for $3 \mathrm{~s}$ before proboscis extension was elicited by applying a droplet of 50\% sucrose solution to each antenna. After proboscis extension the bee was allowed to drink for about $1 \mathrm{~s}$ from that sucrose droplet. Discrimination between CS+ and CSwas tested by presenting each of the odors in random order after each conditioning trial. The time interval between conditioning and presentation of the first odor was $5 \mathrm{~min}$, the second odor was presented $7 \mathrm{~min}$ later. We used this olfactory conditioning protocol because we wanted to apply similar dual choice tests in all three learning experiments. After absolute conditioning to a sensory cue (color or odor) discrimination was tested in dual choice tests. In the olfactory protocol the two odors were offered sequentially. To compare acquisition in different groups of bees, an acquisition score was calculated. This score is the total number of conditioned responses to the $\mathrm{CS}+$ and it has a range between 0 (no conditioned PER) and 7 (conditioned PER after each conditioning trial).

After the last conditioning trial and the following odor tests the bees were fed to satiation with $50 \%$ sucrose solution. They were then transferred with their tubes into a humid chamber kept at room temperature until the retention test on the next day. In the 24-h retention test each odor (CS+ and CS-) was presented to the bee at a temporal interval of $5 \mathrm{~min}$ in random order and it was registered whether PER occurred. As bees were tested repeatedly after the seventh conditioning trial, an extinction phenomenon reducing the PER in the retention test cannot be excluded.

\section{EXPERIMENT 3: COLOR LEARNING WITHOUT SUCROSE REWARD AT THE HIVE ENTRANCE}

In most of the earlier studies visual discrimination at the hive entrance was tested by connecting the hive to two separate entrances. The animals then had to learn that one of the entrances with a specific visual cue gave access to the hive, while the alternative visual cue signaled a blocked entrance. With this paradigm the position of the visual mark signaling the entrance has to be changed frequently to avoid position learning of the cue (Beier and Menzel, 1972; Zhang et al., 2006). We performed an extensive series of pilot experiments over two foraging seasons to define a color learning protocol for the hive entrance. The interference between the experimental bee and other foraging bees returning to the hive can lead to side preferences which are a major problem in these experiments. We also found that experimental bees stop foraging at the feeder when they are tested in dual choice color tests after each visit to the feeder. We developed a hive entrance learning protocol that was similar to the color learning protocol at the food site. In the color learning experiments at the feeder, only the learning color was present during the reward. During an unrewarded test the bee had to choose between the learning color and an alternative. In the hive entrance protocol conditioning and testing was similar to the color learning protocol at the food site. A single bee was conditioned by allowing it to enter the hive marked with a single color plate. In the learning tests the entrance was blocked and the bee could approach and land repeatedly on a plate with the learning color and an alternative.

Similar to the learning protocol at the food site, color learning was tested at the hive entrance in bees which had low or high acceptance thresholds. In this experiment we had to use a small four frame colony to minimize interference between the test bee and other returning foragers of the colony during the choice tests. As the hive entrance was blocked during choice tests, there was no interference with departing bees. Honey bees from the colony were trained to visit a feeder in a small shed $40 \mathrm{~m}$ away from the hive. The bees visiting the feeder were marked individually with colorcoded spots on the thorax and abdomen. The feeder contained $50 \%(\mathrm{w} / \mathrm{w})$ sucrose solution. The experiments were performed with newly alarmed bees that had not visited the feeder before. The acceptance threshold of a newly alarmed bee was determined as described above. During the measurement of the acceptance threshold the hive entrance was open and not marked with a color plate. The concentration of the sucrose solution at the feeder was reduced in steps as described above.

The sucrose thresholds of single bees depend on many exogenous and endogenous factors, like the weather, the state of the hive, the amount of available pollen, the foraging specialization of the bee and its genetic background (Page et al., 2006). The color learning experiments at the food site and the olfactory PER conditioning experiments were performed during the foraging season 2008, while the hive entrance experiments were performed during the season 2009. In all the hive entrance experiments with the 
small four frame colony only one bee had an acceptance threshold of $50 \%$, while many bees had thresholds at $30 \%$. Therefore, bees which accepted sucrose concentrations of $\geq 30 \%$ were assigned to the "high threshold" or "insensitive group" ( $\geq 30 \%$ group). In a number of pilot experiments over several foraging seasons we detected no behavioral differences for learning in the field and in the laboratory between bees that had acceptance thresholds of $\geq 30 \%$ or $\geq 50 \%$. Bees which accepted a concentration of $3 \%$ were assigned to the "low threshold" or "sensitive" group ( $\leq 3 \%$ group). Bees which accepted 10\% but no lower concentrations were not used for the experiments.

After testing the acceptance threshold, an individual bee was allowed to collect $50 \%$ sucrose at the feeder. Other foragers visiting the feeder were caught and kept for the time of the experiment in a small cage. The following experiments were all done with a single bee by two observers. Color choice behavior of the bee was tested when it returned from the feeder to the hive. A white plate $(60 \times 40 \mathrm{~cm})$ was positioned in front of the entrance of a small four frame hive. Bees could enter and leave the hive only through a central tube (diameter $2 \mathrm{~cm}$ ) in the middle of the plate. In the training situation the central tube was open and a colored (yellow or blue) $12 \mathrm{~cm} \times 12 \mathrm{~cm}$ plate surrounding the tube marked the entrance. Whenever the bee returned during the learning phase from the feeder to the hive it could enter the hive entrance which was marked by the plate with the learning color. In the test situation the central tube was covered with a white plate so that the returning bees could not enter the hive anymore. A yellow and a blue colored square plate was positioned $24 \mathrm{~cm}$ to the right and to the left of the center tube. The plates had a dark round center (diameter $2 \mathrm{~cm}$ ) which looked like the hive entrance during the training phase. Opaque Plexiglas ${ }^{\circledR}$ GS (3-mm thick; colors "yellow" or "sky blue"; www.modulor.de) was used for the colored plates during training and testing. To avoid odor cues, different plates were used during training and testing and all plates were cleaned every day with ethanol.

Behavior of an individual bee in the choice situation at the hive was recorded with two video cameras (Sony HDR-CX11E) positioned approximately $200 \mathrm{~cm}$ from the right and the left colored plate during the test situation. The focal length of each camera was adjusted to record the area of the color plate and approximately $1 \mathrm{~cm}$ around the plate. Behavior of the bee during the choice test was recorded for $3 \mathrm{~min}$, then the positions of the two plates were exchanged and behavior was recorded for another $3 \mathrm{~min}$. This test procedure helps to avoid artifacts due to a side preference of the bee. The videos were later analyzed in the slow motion mode and when necessary in the single frame mode. The test bee could be easily distinguished by the color dots on the thorax and abdomen from other foragers of the colony that also returned to the hive. As we used a small colony, there were not many other bees returning to the hive during the color tests. "Approaches" toward each of the two alternative plates were counted for the two tests each lasting $3 \mathrm{~min}$. An "approach" toward the plate was registered whenever the individual test bee was flying in front of the color plate. In addition, the "time" spent hovering in front of a plate or spent sitting on a plate was measured. The times for each plate were added for the two $3 \mathrm{~min}$ tests. The proportions of approaches and time between the two alternative plates were calculated and used as measures indicating choice behavior between the two alternatives.
After assigning a single bee to the low or high acceptance threshold group, the learning experiment started. At the beginning of the experiment spontaneous color choice behavior was tested by closing the hive entrance in the middle and offering the bee the two alternative color plates right and left of the entrance. The bee could not enter the hive through the two color plates. Behavior was recorded for $6 \mathrm{~min}$, after $3 \mathrm{~min}$ the positions of the plates were exchanged. The initial positions of the two plates were chosen at random. Then the two test plates were removed and a yellow plate was mounted at the hive entrance which was now open again. The test bee was allowed to collect 50\% sucrose at the feeder and to return 10 times to the hive through the yellow plate covering the hive entrance. After 10 visits to the feeder and the hive the entrance was closed again and choice behavior of the individual bee was recorded as described above for the spontaneous behavior. Again, the initial positions of the two plates were chosen at random.

Small differences of learning performance in bees are often difficult to detect if only one alternative is conditioned. In tactile conditioning experiments with pollen and non-pollen bees it was shown that minor differences in acquisition and discrimination can be detected with a reversal learning protocol in which the bee is first conditioned to one alternative and then to the other (Scheiner et al., 1999). From a number of pilot experiments at the hive entrance we knew that there exist only small differences in acquisition and discrimination between bees with different acceptance thresholds. Therefore, we used a reversal learning protocol for hive entrance conditioning. After this first learning test the hive entrance was marked by a blue plate to test reversal learning. As before, the bee could visit the feeder and enter the hive 10 times through the blue plate covering the entrance. Choice behavior was tested again for $2 \times 3$ min after 10 visits to the hive.

The behavior of 15 bees in each of the two acceptance threshold groups was analyzed in the three behavioral tests before learning, after entering the hive 10 times through the yellow plate and after entering the hive 10 times through the blue plate. After the tests with an individual bee, the foraging group, which was kept in a cage, was set free and could forage again at the feeder. During that time the hive entrance was not marked by a color plate. As other bees of the colony could enter the hive during the training sessions of an individual bee, only one learning experiment was performed during a day and a new experiment started on the next day. As bees take about $5 \mathrm{~min}$ for a round trip between feeder and hive, one can estimate that individual bees of the colony use the unmarked hive entrance over 100 times during the approximately $10 \mathrm{~h}$ of foraging after the previous experiment. It cannot be excluded that a newly alarmed test bee had entered the hive entrance with the blue or yellow conditioning plate on the day before. We minimized this effect by performing only one color learning experiment per day and by keeping the hive entrance open without a color plate for most hours of the day.

\section{STATISTICS}

\section{Color learning at a food site}

The proportions of approaches toward the green and blue alternatives were transformed using a modification of the arcsin(sqrt) function (Freeman and Tukey, 1950; Zar, 1999). The transformed data were tested for significant deviations from normal distribution 
$(p<0.05$; D'Agostino and Pearson omnibus normality test; GraphPad Prism 4). No significant deviations from normal distributions were found. Therefore, parametric methods were used for statistic comparisons. Data of learning trials and the 24-h retention test were statistically compared between individuals of each acceptance groups using a two way ANOVA with repeated measures (GraphPad Prism 4).

\section{Olfactory PER conditioning}

Acquisition scores and GRSs for each sucrose acceptance group ( $\geq 50 \%$ or $\leq 3 \%$ ) were tested for significant deviations from normal distributions ( $p<0.05$; D'Agostino and Pearson omnibus normality test; GraphPad Prism 4). Most groups were not distributed normally. Therefore, medians, quartiles and mean values are presented in the figures. Statistic differences of acquisition scores and GRS between the two sucrose acceptance groups were tested with the Mann-Whitney U test (GraphPad Prism 4). Differences in the $24 \mathrm{~h}$ retention test between the acceptance groups were analyzed using Fisher's exact probability test (GraphPad Instat 3.06). Statistic differences between the acquisition curves for both acceptance groups were analyzed using a binary logistic regression model for repeated measurements (Wright et al., 2007). Statistics were calculated with the GENLIN command of the statistic software PASW Statistics 18, using a binomial distribution, logit linking function and repeated measurements.

\section{Hive entrance color learning}

For statistical analyses proportions of times spent on the yellow or blue alternative were transformed using the $\arcsin (\operatorname{sqrt}(\mathrm{p}))$ function (Zar, 1999). Proportions of approaches were transformed using a modification of the arcsin(sqrt) function (Freeman and Tukey, 1950; Zar, 1999). Data were tested for deviations from normal distributions $(p<0.05$; D'Agostino and Pearson omnibus normality test; GraphPad Prism 4). No significant deviations from normal distributions were found.

\section{RESULTS \\ COLOR LEARNING AT A FOOD SITE IN THE FIELD}

In the first experiment free flying nectar foragers with high and low sucrose acceptance thresholds $(\geq 50 \%$ and $\leq 3 \%)$ were tested in a color learning protocol at a food site in the field. The acquisition curves and retention after $24 \mathrm{~h}$ for bees from the two acceptance groups are shown in Figure 1. The acquisition functions clearly differ between bees with acceptance thresholds $\leq 3 \%$ and $\geq 50 \%$. Bees of the $3 \%$ acceptance group show faster acquisition because the choice reaction differs significantly already after the first learning trial from spontaneous choice (Bonferroni posttest; $p<0.001$ ), while bees from the $50 \%$ group reach this level of significance after the third learning trial. After 3-5 rewards bees of both groups reach stable acquisition plateaus. At the plateau there exist no more statistic differences between consecutive choice tests (Bonferroni posttests; $p>0.05 ; 50 \%$ acceptance after the third learning trial; $3 \%$ acceptance after the fifth learning trial). Highly significant differences of the choice reactions between the two groups were found for each single learning trial (unpaired $t$-test, $p<0.001)$. A two way ANOVA for repeated measures shows that acceptance $(F=64.02$, DF: $1,304, p<0.0001)$ and the learning

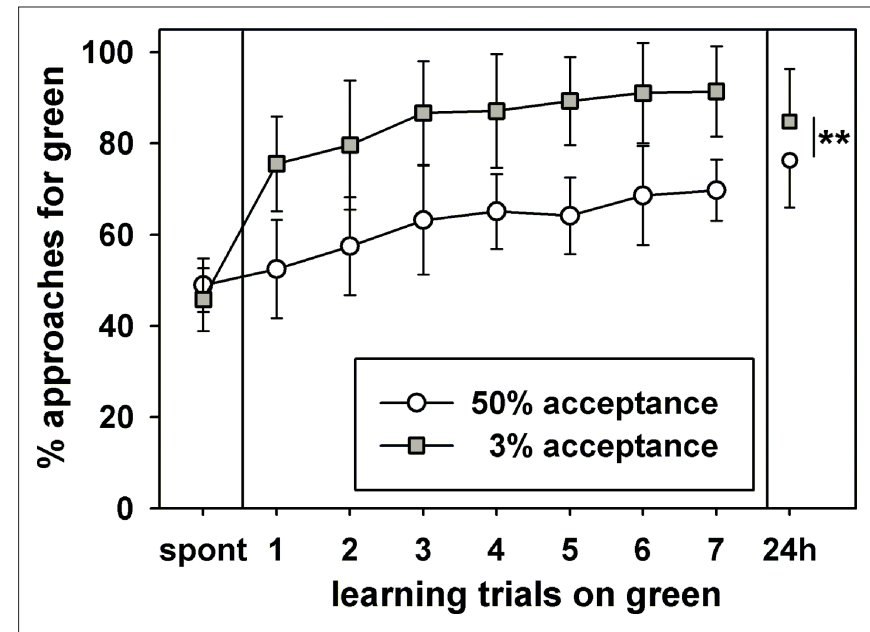

FIGURE 1 | Color learning experiment at an artificial feeder in the field for bees which either had acceptance thresholds $\leq 3 \%$ or $\mathbf{2 5 0} \%$. The bees could choose between a green and blue alternative (for details see text). The abscissa shows the spontaneous choice (spont), choice tests after seven

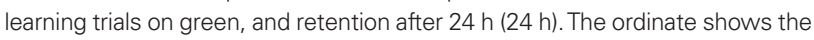
mean percentages of approaches with the respective standard deviations for the rewarded green alternative. The stars indicate significant differences of the choice reaction in the 24-h retention test (2-sided t-test, $p<0.01$ ); other significant differences are not indicated in the figure, for details see text. 20 bees were tested in each group. Bees in the $50 \%$ acceptance group made between 4 and 17 approaches in the tests, resulting in a total of 3143 approaches for this group. Bees in the $3 \%$ acceptance group made between 5 and 17 approaches in the tests, resulting in a total of 3370 approaches for this group.

trials $(F=58.83$, DF: $8,304, p<0.0001)$ have significant effects. Also the interactions are highly significant $(F=14.03$, DF: 8,304 , $p<0.0001)$.

In the retention test after $24 \mathrm{~h}$ the percentages of approaches toward the conditioned color were significantly different between the two acceptance groups ( 2 -sided $t$-test, $p<0.01$ ). It is remarkable that the choice reaction of the $3 \%$ acceptance group at $24 \mathrm{~h}$ was significantly reduced compared to the seventh learning trial (Bonferroni posttest; $p<0.001$ ), while for the $50 \%$ acceptance group the percentage of correct choices in the retention test at $24 \mathrm{~h}$ was significantly larger than the choice reaction after the seventh trial (Bonferroni posttest; $t=2.374, p<0.05$ ). These findings demonstrate a convergence of choice performance but still significant differences between the two acceptance groups in the 24-h retention test.

\section{PER RESPONSIVENESS, OLFACTORY PER CONDITIONING AND 24H RETENTION IN THE LABORATORY}

The olfactory PER conditioning experiments in the laboratory were done with bees that had either $\geq 50 \%$ or $\leq 3 \%$ sucrose acceptance in the field. At the beginning of the laboratory experiment PER responsiveness for different sucrose concentrations was tested in these bees to compare sucrose acceptance in the field with sucrose PER responsiveness in the laboratory. The sucrose concentration dependent PER curves for the two acceptance groups were very similar and the two GRSs which are a compound measure for responsiveness were not significantly different (Figure 2; Mann-Whitney 
$\mathrm{U}$ Test, $p>0.05)$. We conclude from this experiment that the bees in the two sucrose acceptance groups show similar sucrose responsiveness in the laboratory.
Acquisition curves, discrimination and 24-h retention tests for bees from the two acceptance groups are shown in Figure 3A. Bees from the $\leq 3 \%$ acceptance group clearly show better acquisition and
A

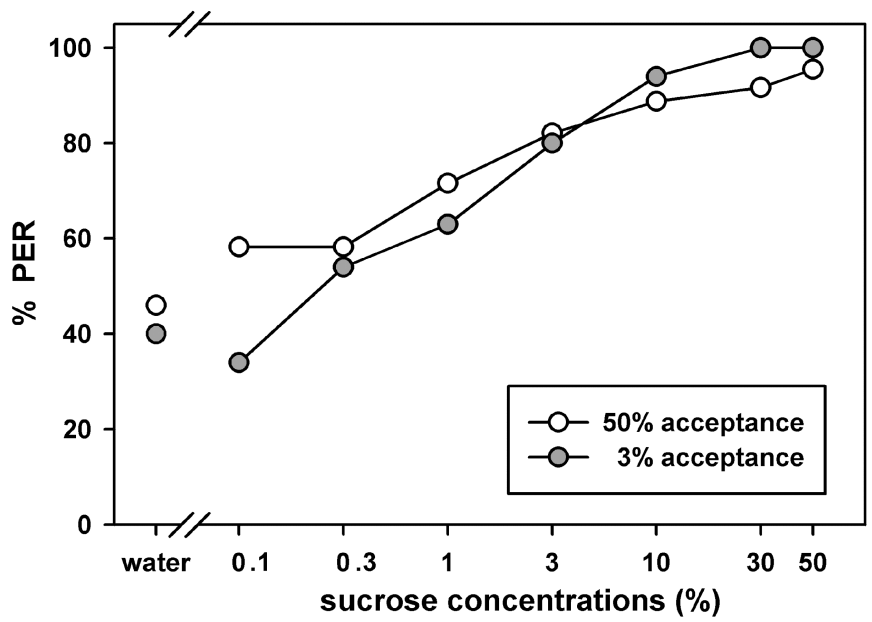

B

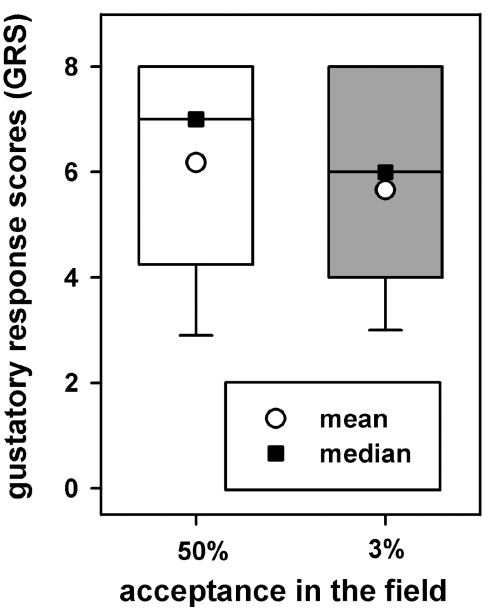

FIGURE 2 | PER responsiveness for different concentrations of sucrose in two groups of bees which either had acceptance thresholds $\leq 3 \%$ or $\geq 50 \%$ in the field. (A) Sucrose concentration dependence of the PER. The abscissa shows the applied stimuli starting from water to $50 \%$ sucrose. The ordinate shows the percentages of PER for each stimulus. (B) The gustatory response scores for the two groups (for details see text). The box plots show medians, means, quartiles, the whiskers indicate $95 \%$ percentiles. 35 bees were tested in the $3 \%$ acceptance group and 28 bees in the $50 \%$ acceptance group.
A

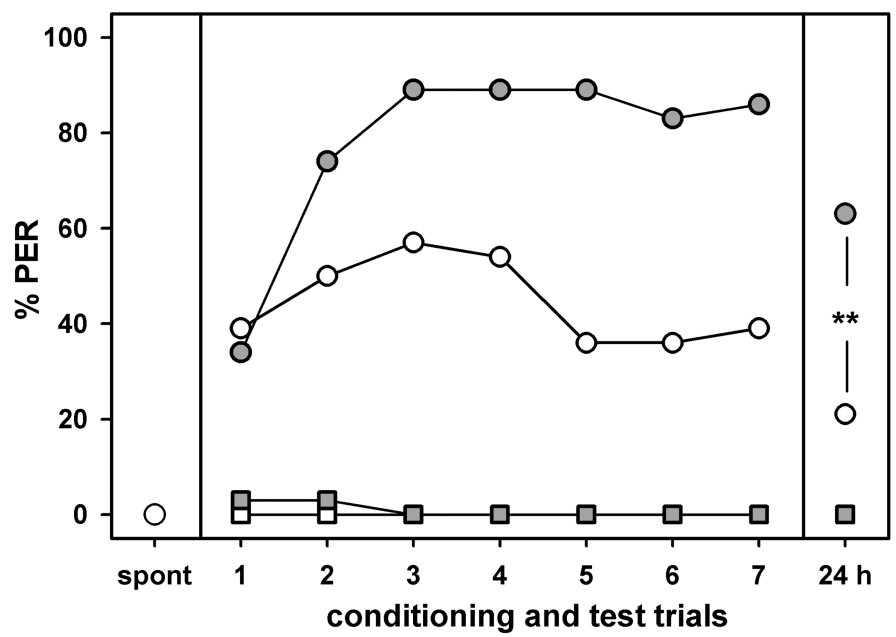

$-0-50 \%$ acceptance $\mathrm{CS}+$

$-\square-50 \%$ acceptance CS -

-O- $3 \%$ acceptance CS +

$\rightarrow-3 \%$ acceptance CS -
B

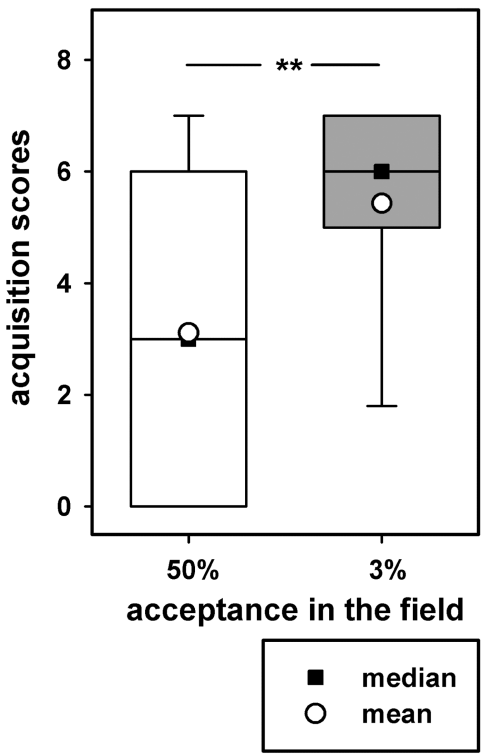

FIGURE 3 | Olfactory PER conditioning for two groups of bees which either had acceptance thresholds $\leq 3 \%$ or $\mathbf{2 5 0} \%$ in the field. (A) The acquisition curves, discrimination, and $24-h$ retention in the two groups. The abscissa shows spontaneous choice (spont), tests after each of the seven learning trials, and 24-h retention (24h). The ordinate shows the percentages of PER to the conditioned odor cineol (CS+) and the alternative not rewarded odor clove oil (CS-). ${ }^{* *}$ indicates a significant difference for the retention tests
(Fischer's exact probability test, $p<0.01$ ); other significant differences in the acquisition curves are not indicated (for details see text). (B) The acquisition scores for the two groups (for details see text). The box plots show medians, means, quartiles, the whiskers show $95 \%$ percentiles. ${ }^{* *}$ indicates a significant difference between acquisition scores (Mann-Whitney UTest, $p<0.01$ ). 35 bees were tested in the $3 \%$ acceptance group and 28 bees in the $50 \%$ acceptance group. 
retention than bees from the $\geq 50 \%$ acceptance group. No differences in odor discrimination were found between bees of the two groups. Compared to spontaneous behavior, bees of both groups show significant increase of conditioned PER after the first conditioning trial (Fisher exact probability test, $p<0.001$ ). The acquisition curves differ significantly between the two acceptance groups (binary logistic regression model for repeated measurements, Wald-chi-square $=12.89, \mathrm{df}=1, p<0.001)$. As a consequence of the differences in acquisition also the acquisition scores which are compound measures of acquisition differ significantly (Figure 3B; Mann-Whitney $\mathrm{U}$ test, $p<0.01$ ). The differences in acquisition between the two sucrose acceptance groups are similar to those for color learning with free flying bees from the same acceptance groups (Figure 1). Apparently, differences in sucrose acceptance in the field are also correlated with differences in olfactory PER conditioning in the laboratory, although sucrose responsiveness measured with the PER protocol in the laboratory does not differ between the two groups (Figure 2). In the 24-h retention tests the percentages of conditioned responses for both groups were lower compared to the response behavior after the seventh conditioning trial but these differences were not significant (Fisher exact probability test, $p>0.05)$. Similar to the results during the conditioning phase, the differences of conditioned PER between the two sucrose acceptance groups were significant also in the 24 -h retention tests (Fisher exact probability test, $p<0.01$ ). We conclude from these experiments that differences of sucrose acceptance in the field are correlated with differences in acquisition and retention in olfactory conditioning in the laboratory.

A

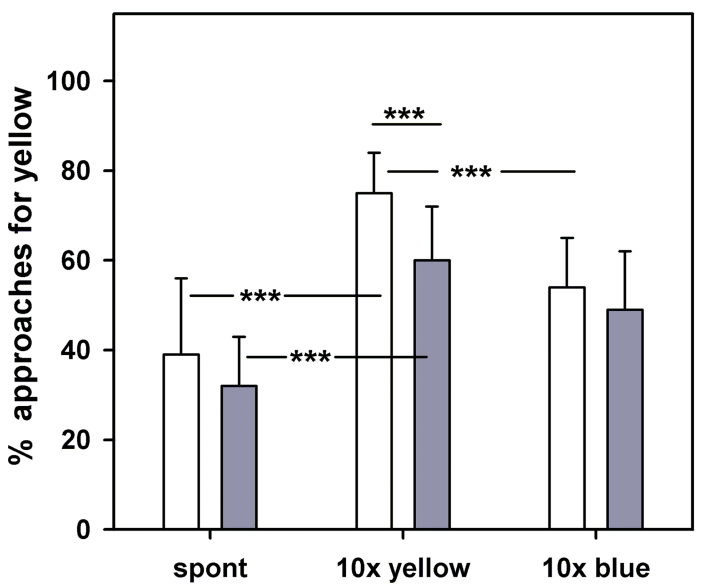

\section{HIVE ENTRANCE COLOR LEARNING}

The experiments were done with individual bees which either had sucrose acceptance thresholds $\geq 30 \%$ or $\leq 3 \%$. Bees of the two groups did not differ significantly in the mean times for a roundtrip from feeder to hive and back (30\% group: $5.2 \pm 0.49 \mathrm{~min}$; $3 \%$ group: $4.7 \pm 0.23 \mathrm{~min} ; 2$ sided $t$-test, $p=0.43$ ).

To quantify color choice behavior we measured approaches toward the yellow and blue plates and the time spent in front of the plates or on the plates. In all tests the percentages of approaches and of time were very similar (Figures 4 A,B $)$. Approaches and times are significantly correlated for both experimental groups when spontaneous choice tests, tests after 10 learning trials on yellow and on blue are used for calculating the correlation (Pearson correlation; 30\% group: $r=0.8388, p<0.0001 ; 3 \%$ group: $r=0.6585$, $p<0.0001)$. Also the results of the statistical comparisons within each group and between different phases of the experiment are very similar for the two behavioral measures. The results demonstrate that the two behavioral measures used for the hive entrance learning experiments are closely correlated.

Spontaneously bees of both groups approach the yellow plate less frequently than the blue plate (Figures 4A,B; one sample $t$-test, tested against a theoretical value of $50 \%$ choices for yellow; 30\% acceptance group: $p<0.05 ; 3 \%$ acceptance group: $p<0.0001)$. Similar to the approaches, bees of both groups also spend less time on the yellow alternative. Bees of the 3\% acceptance group spend significantly less time on the yellow alternative, while this difference is not significant for the 30\% group (one sample $t$-test, tested against a theoretical value of $50 \%$ time
B

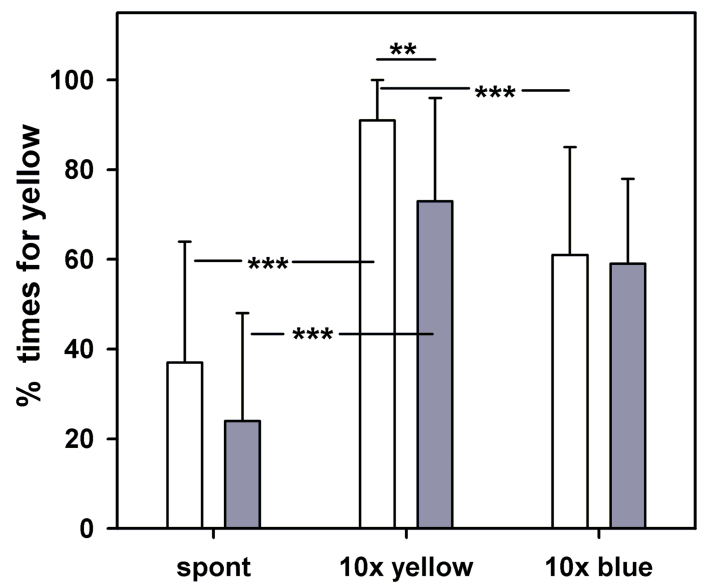

$30 \%$ acceptance $3 \%$ acceptance

FIGURE 4 | Color learning at the hive entrance for two groups of bees which had acceptance thresholds $\leq 3 \%$ or $\mathbf{2 3 0} \%$ in the field. The abscissa of both graphs show choice behavior before learning (spont), after 10 learning trials with a yellow plate marking the hive entrance (10× yellow), and after 10 reversal learning trials with a blue plate marking the hive entrance (10x blue). (A)The ordinate shows mean percentages of approaches and the respective standard deviations toward the yellow plate compared with the approaches toward a blue plate (for details see text). (B) The ordinate shows mean percentages of times and the respective standard deviations spent in front or on the yellow plate compared with the times for a blue plate (for details see text). Significant differences of Bonferroni multiple comparison tests (for comparisons within an acceptance group) and of 2-sided t-tests (between acceptance groups) are indicated $\left({ }^{* *} p<0.01 ;{ }^{* *} p<0.001\right.$; for details see text). Fifteen bees were tested in each of the two acceptance groups. 
spent on yellow; $30 \%$ acceptance group: $p>0.05 ; 3 \%$ acceptance group: $p<0.001)$. The differences in spontaneous choice both for approaches and times between the two acceptance groups are not statistically significant ( $t$-tests; approaches: $p>0.05$; time: $p>0.05)$. After 10 learning trials using the yellow entrance plate, the percentages of approaches and of time for the yellow alternative are significantly enhanced for bees of both sucrose acceptance groups (Figures 4A,B; Bonferroni multiple comparison tests, $p<0.001)$. After 10 learning trials on yellow, bees of the $3 \%$ group show significantly lower percentages of approaches and times for the yellow alternative than the $30 \%$ group (Figures $4 \mathbf{A}, \mathbf{B}$; $t$-tests, approaches: $p<0.001$; times: $p<0.01)$. After reversal learning with the blue entrance plate, bees of the $30 \%$ sucrose acceptance group show highly significant reductions of approaches and times for the yellow alternative (Figures 4A,B; Bonferroni multiple comparison tests, $p<0.001)$. The reductions of approaches and time for the yellow alternative after reversal learning are not significant for the $3 \%$ group (Figures 4A,B; Bonferroni multiple comparison tests, $p>0.05)$. We conclude from these experiments that the differences in acquisition that we found for sucrose dependent learning in the field and in the laboratory are not apparent in a learning protocol without sucrose rewards. Bees from the sensitive sucrose acceptance group (acceptance threshold $\leq 3 \%$ ) do not show better acquisition than bees from the insensitive group (acceptance threshold $\geq 30 \%$ ), quite contrarily, reversal color learning is better in the insensitive bees compared to the sensitive sucrose acceptance group.

\section{DISCUSSION}

With our experiments we have tested a number of hypotheses concerning the relations between sensitivity for sucrose and learning. We know now that some of the rules developed in laboratory experiments are valid also in the field and that sucrose acceptance in the field is a sensitive parameter to predict learning performance and retention also in the laboratory. Furthermore we have shown for the first time that the relations between sucrose sensitivity and learning only apply to protocols in which sucrose serves as a reward.

Before we performed the present study, learning experiments in the laboratory using different olfactory, tactile and operant protocols (Erber et al., 1998; Scheiner et al., 1999, 2001a,b, 2003, 2005) led to the hypothesis that high sucrose sensitivity is correlated with better acquisition and retention also in the field. The experiments discussed here support this hypothesis. Nectar foraging bees which accept low sucrose concentrations when visiting an artificial food site show better acquisition and 24 -h retention in a color learning assay than bees which accept only high sucrose concentrations.

Generally it is not easy to compare experiments with bees in the field with those in the laboratory because the analyzed behaviors are not identical. The estimation of sucrose sensitivity is a good example for these difficulties. Sucrose sensitivity in the laboratory is determined by stimulating bees with different concentrations of sucrose and by registering at which concentrations an individual bee extends its proboscis. The PER is a relatively simple reaction to a gustatory stimulus. The sucrose sensitivity estimated with this experiment is valid for a stimulus-response relationship under laboratory conditions. In the field sucrose sensitivity is estimated by measuring which sucrose concentrations a bee is willing to accept during foraging. Sucrose acceptance in the field is the result of a complex behavioral sequence in which a bee first has to land on a food site; second it has to probe the offered sucrose solution with the antennae which can lead to extension of the proboscis. At the end of this sequence a bee can either accept the sucrose solution or it can search for another food source (Mujagic and Erber, 2009). In addition, the behavioral contexts between free flying and harnessed bees are completely different which can affect sensory thresholds if the same bee is tested in the field and in the laboratory. Similar arguments can be applied to the different learning protocols in the field and in the laboratory.

Our experiments demonstrate that sucrose acceptance is a sensitive measure of gustatory thresholds and that the measure of PER responsiveness is less sensitive in nectar foragers that accept different sucrose concentrations when foraging in the field. The thresholds of bees accepting low sucrose concentrations $(\leq 3 \%)$ differed from the thresholds of bees accepting high sucrose concentrations $(\geq 50 \%)$ by approximately $1.2 \mathrm{log}$ units of concentration, still the PER concentration-response curves of the same individuals were very similar under laboratory conditions. The experiments also demonstrate that differences of learning performance in olfactory PER conditioning can exist even in bees which show the same PER responsiveness in the laboratory. Earlier studies showed that significant differences in PER responsiveness in the laboratory were correlated with learning performance in tactile or olfactory PER conditioning (Erber et al., 1998; Scheiner et al., 1999, 2001a,b, 2003, 2005). Meanwhile, additional laboratory experiments prove that differences in PER responsiveness are not a necessary prerequisite for differences in PER conditioning. Also other factors like the social role, foraging specialization, foraging age and the state of health (Behrends et al., 2007; Iqbal and Müeller, 2007; Drezner-Levy et al., 2009; Scheiner and Amdam, 2009); can affect learning. In an olfactory PER conditioning experiment with bees that had identical sucrose PER responsiveness, Behrends et al. (2007) demonstrated that old foragers showed inferior learning performance compared to younger foragers. The decrease in learning performance seems not to be related to the chronological age of the animals, but rather to their foraging age and specialization in the field (Behrends et al., 2007; Scheiner and Amdam, 2009). Sucrose acceptance in the field probably is the better predictor for learning performance because this measure is closely related to the foraging specialization in the field (Mujagic and Erber, 2009).

The present experiments clearly show that the individual evaluation of the reward is decisive for learning performance in different protocols that use sucrose as a reward in the field and in the laboratory. Earlier studies showed that associative learning performance in bees is strongly correlated with the concentration of the sucrose reward and that higher sucrose concentrations improve learning performance (Loo and Bitterman, 1992; Couvillon et al., 1994). Laboratory experiments demonstrated that the individual threshold for sucrose and the sucrose concentration in tactile PER conditioning determine the individual value of the reward (Scheiner et al., 1999). By adjusting the reward concentration to the sensory responsiveness of an individual, equal subjective rewards can be generated which lead to similar acquisition and retention in bees that differ significantly in sucrose responsiveness (Scheiner et al., 2005). All these experiments suggest that differences in the perception of sucrose lead to differences in acquisition and retention. 
The results of our color experiments at the food site are in good accordance with the learning experiments in the laboratory. Although color learning at a food site and olfactory PER conditioning in the laboratory are two very different forms of associative learning in the bee, the same basic rules between sucrose sensitivity and learning are valid. Bees which accept low sucrose concentrations show better acquisition and retention compared to bees which accept only higher sucrose concentrations. These rules become apparent when the bees with different sucrose sensitivity are rewarded with a high sucrose concentration (50\%). Together with the earlier experiments, these results suggest that the subjective reward value is stronger in sucrose sensitive bees than in insensitive bees (Scheiner et al., 2005). Associative learning in the field is dependent on a number of exogenous and endogenous factors which influence learning performance by modulating sucrose sensitivity. The foraging specialization of an individual, the season, the availability of nectar and pollen, the weather and the complex conditions within the colony affect the individual sucrose sensitivity of a foraging bee (for a discussion see Page et al., 2006) and hence learning. We think that the existing data can now be used for modeling the consequences of these different factors for learning under natural conditions.

The hive entrance color learning experiments clearly show that the differences in associative learning which can be found in bees with different sucrose acceptance vanish when sucrose does not represent the reward. This finding supports the hypothesis that differences in associative learning performance and retention in bees are causally related to differences in subjective reward evaluation. Contrary to the learning experiments with sucrose rewards, the results with hive entrance conditioning indicate that acquisition and reversal learning is better for bees which accept only high sucrose concentrations $(\geq 30 \%)$. At the moment we do not have a hypothesis concerning these differences. The close relationships between the percentages of approaches and the percentages of time spent in front of the color plates suggest that both measures are robust indicators of color choice. It is possible that the subjective

\section{REFERENCES}

Barker, P. D. R., and Lehner, Y. (1974). Acceptance and sustenance value of naturally occurring sugars fed to newly emerged adult workers of honey bees (Apis mellifera L.). J. Exp. Zool. 187, 277-285.

Behrends, A., Scheiner, R., Baker, N., and Amdam, G. V. (2007). Cognitive aging is linked to social role in honey bees (Apis mellifera L.). Exp. Gerontol. 42, 1146-1153.

Beier, W., and Menzel, R. (1972). Untersuchungen über den Farbensinn der deutschen Wespe (Paravespula germanica F., Hymenoptera, Vespidae): Verhaltensphysiologischer Nachweis des Farbensehen. Zool. Jb. Physiol. 76, 441-454.

Bitterman, M.E., Menzel, R., Fietz,A., and Schäfer, S. (1983). Classical conditioning of proboscis extension in honeybees (Apis mellifera). J. Comp. Psychol. 97, 107-119.
Chittka, L., and Menzel, R. (1992). The evolutionary adaptation of flower colours and the insect pollinators' colour vision. J. Comp. Physiol. A. 171, 171-181.

Couvillon, P. A., Nagrampa, J. A., and Bitterman, M. E. (1994). Learning in honeybees (Apis mellifera) as a function of sucrose concentration: analysis of the retrospective effect. J. Comp. Psychol. 108, 274-281.

Drezner-Levy, T., Smith, B., and Shafir, S. (2009). The effect of foraging specialization on various learning tasks in the honeybee (Apis mellifera). Behav. Ecol. Sociobiol. 64, 135-148.

Erber, J. (1975a). Dynamics of learning in honey bee (Apis mellifica carnica). 1. Time dependence of choice reaction. J. Comp. Physiol. A. 99, 231-242.

Erber, J. (1975b). Dynamics of learning in honey bee (Apis mellifica carnica). 2. Principles of information processing. J. Comp. Physiol. A. 99, 243-255.

reward values of bees with different acceptance thresholds are different. One factor affecting the subjective reward value in the hive could be the delivery time of the collected sucrose (Lindauer, 1948; Seeley, 1995). As both groups were fed the same sucrose concentration (50\%) at the feeder and as the times for roundtrips between food site and hive did not differ between the two groups, this hypothesis is not supported by the experimental data. Clearly, more experiments are necessary to find out in which additional behavioral traits bees with low and high sucrose acceptance thresholds differ. A large number of experiments demonstrate that foraging specialization in bees is correlated with differences in genetic architecture, in phenotypic architecture and in signaling cascades which in turn can affect sensory processing and stimulus evoked responses (Page et al., 2006). There remain many open questions concerning the division of foraging labor and associative learning in the field. Future experiments under natural conditions comparing learning of bees with different foraging specializations are necessary to understand the relations between sensory sensitivity and learning. It would be most interesting to perform a learning experiment comparing water foragers with pollen- and nectar foragers. So far we know that the differences in sucrose acceptance in free flying nectar foragers have effects on associative learning in which sucrose is the reward. For a better understanding of the complex interactions between sensory sensitivity and learning in the field we need similar experiments with foraging bees that collect water or pollen.

\section{ACKNOWLEDGMENTS}

The work was supported by the Deutsche Forschungsgemeinschaft by funding of the Graduate College "Functional Insect Science". We wish to thank Benedikt Polaczek for his advice and support in beekeeping. We thank Joachim Buchholz for excellent technical support in building the experimental setups and for his help in performing and evaluating the hive learning experiments. The experiments comply with the current German guidelines for the proper treatment of animals.

Erber, J., Kierzek, S., Sander, E., and Grandy, K. (1998). Tactile learning in the honeybee. J. Comp. Physiol. A. $183,737-744$.

Freeman, M. F., and Tukey, J. W. (1950). Transformations related to the angular and the square root. Ann. Math. Statist. 21, 607-611.

Frings, H. (1944). The loci of olfactory end organs in the honey bee, Apis mellifera Linn. J. Exp. Zool. 97, 123-134.

Harris, R.A., de Ibarra, N. H., Graham, P., and Collett, T. S. (2005). Ant navigation: priming of visual route memories. Nature 438, 302.

Hori, S., Takeuchi, H., Arikawa, K., Kinoshita, M., Ichikawa, N,. Sasaki, M., and Kubo, T. (2006). Associative visual learning, color discrimination, and chromatic adaptation in the harnessed honeybee (Apis mellifera L.). J. Comp. Physiol. A. 192, 691-700.

Iqbal, J., and Müeller, U. (2007). Virus infection causes specific learning deficits in honeybee foragers. Proc. $R$. Soc. Lond. B 274, 1517-1521.

Kisch, J., and Erber, J. (1999). Operant conditioning of antennal movements in the honey bee. Behav. Brain Res. 99, 93-102.

Kisch, J., and Haupt, S. S. (2009). Side specific operant conditioning of antennal movements in the honey bee. Behav. Brain Res. 196, 131-133.

Lindauer,M.(1948).Ueber dieEinwirkung von Duft- und Geschmacksstoffen sowie anderer Faktoren auf die Taenze der Bienen. J. Comp. Physiol. A. 31, 348-412.

Loo, S. K., and Bitterman, M. E. (1992). Learning in honeybees (Apis mellifera) as a function of sucrose concentration. J. Comp. Psychol. 106, 29-36.

Masuhr, Th., and Menzel, R. (1972). "Learning experiments on the use of side specific information in the olfactory and visual system in the honeybee (Apis mellifica)," in Information 
Processing in the Visual Systems of Arthropods, ed. R. Wehner (Berlin, Heidelberg, New York: Springer Verlag), 315-322.

Menzel, R. (1967). Untersuchungen zum Erlernen von Spektralfarben durch die Honigbiene (Apis mellifica). J. Comp. Physiol. A. 56, 22-62.

Menzel, R. (1969). Das Gedaechtnis der Honigbiene fuer Spektralfarben. J. Comp. Physiol. A. 63, 290-309.

Menzel, R., and Müller, U. (1996). Learning and memory in honeybees: From behavior to neural substrates. Annu. Rev. Neurosci. 19, 379-404.

Mujagic, S. (2009). Sensorische Empfindlichkeiten, Lernen und Sammelverhalten bei der Honigbiene (Apis mellifera L.) (Technische Universität Berlin: Dissertation) URN: nbn:de:kobv:83-opus-22824.

Mujagic, S., and Erber, J. (2009). Sucrose acceptance, discrimination and proboscis responses of honey bees (Apis mellifera L.) in the field and the laboratory. J. Comp. Physiol. A. 195, 325-339.

Niggebrügge, C., Leboulle, G., Menzel, R., Komischke, B., and de Ibarra, N. H. (2009). Fast learning but coarse discrimination of colours in restrained honeybees. J. Exp. Biol. 212, 1344-1350.

Núñez, J. A. (1966). Quantitative Beziehungen zwischen den Eigenschaften von Futterquellen und dem Verhalten von Sammelbienen. J. Comp. Physiol. A. 53, 142-164.

Page, R., and Erber, J. (2002). Levels of behavioral organization and the evolution of division of labor. Naturwissenschaften 89, 91-106.

Page, R. E., Erber, J., and Fondrk, M. K. (1998). The effect of genotype on response thresholds to sucrose and foraging behavior of honey bees (Apis mellifera L.). J. Comp. Physiol. A. 182, 489-500.

Page, R. E., Scheiner, R., Erber, J., and Amdam, G. V. (2006). The development and evolution of division of labor and foraging specialization in a social insect (Apis mellifera L.). Curr. Top. Dev. Biol. 74, 253-286.

Pankiw, T., and Page, R.E. (2000). Response thresholds to sucrose predict foraging division of labor in honeybees. Behav. Ecol. Sociobiol. 47, 265-267.

Scheiner, R. (2004). Responsiveness to sucrose and habituation of the proboscis extension response in honey bees. J. Comp. Physiol. A. 190, 727-733.

Scheiner, R., and Amdam, G. V. (2009). Impaired tactile learning is related to social role in honeybees. J. Exp. Biol. 212, 994-1002.

Scheiner, R., Barnert, M., and Erber, J. (2003).Variation in water and sucrose responsiveness during the foraging season affects proboscis extension learning in honey bees. Apidologie (Celle) 34, 67-72.

Scheiner, R., Erber, J., and Page, R. E. (1999). Tactile learning and the individual evaluation of the reward in honey bees (Apis mellifera L.). J. Comp. Physiol. A. 185, 1-10.

Scheiner, R., Kuritz-Kaiser, A., Menzel, R., and Erber, J. (2005). Sensory responsiveness and the effects of equal subjective rewards on tactile learning and memory of honeybees. Learn. Mem. 12, 626-635.

Scheiner, R., Page, R. E., and Erber, J. (2001a). Responsiveness to sucrose affects tactile and olfactory learning in preforaging honey bees of two genetic strains. Behav. Brain Res. 120, 67-73.

Scheiner, R., Page, R. E., and Erber, J. (2001b). The effects of genotype, foraging role, and sucrose responsiveness on the tactile learning performance of honey bees (Apis mellifera L.). Neurobiol. Learn. Mem. 76, 138-150.

Scheiner, R., Page, R. E., and Erber, J. (2004). Sucrose responsiveness and behavioral plasticity in honey bees (Apis mellifera). Apidologie (Celle) 35, 133-142.

Schremmer, F. (1941). Versuche zum Nachweis der Rotblindheit von Vespa rufa L. J. Comp. Physiol. A 28, 457-466.

Seeley, T. D. (1995). The Wisdom of the Hive. Cambridge, London: Harvard University Press.

Vareschi,E.(1971).Duftunterscheidungbei der Honigbiene. Einzelzellableitungen und Verhaltensreaktionen. J. Comp. Physiol. A. 75, 143-173.

von Frisch, K. (1927). Versuche ueber den Geschmackssinn der Bienen. Naturwissenschaften 15, 321-327.

von Frisch, K. (1965). Tanzsprache und Orientierung der Bienen. Berlin, Heidelberg, New York: Springer Verlag.

Waser, N. M. (1986). Flower constancy: definition, cause, and ceasurement. Am. Nat. 127, 593-603.
Wright, G. A., Mustard, J. A., Kottcamp, S. M., and Smith, B. H. (2007). Olfactory memory formation and the influence of reward pathway during appetitive learning by honey bees. J. Exp. Biol. 210, 4024-4033.

Zar, J. H. (1999). Biostatistical Analysis. Polynomial Regression. 4th Edn. Upper Saddle River, NJ: Prentice Hall.

Zhang, S., Schwarz, S., Pahl, M., Zhu, H., and Tautz, J. (2006). Honeybee memory: a honeybee knows what to do and when. J. Exp. Biol. 209, 4420-4428.

Conflict of Interest Statement: The authors declare that the research was conducted in the absence of any commercial or financial relationships that could be construed as a potential conflict of interest.

Received: 12 April 2010; paper pending published: 14 May 2010; accepted: 29 June 2010; published online: 19 July 2010.

Citation: Mujagic S, Sarkander J, Erber

$B$ and Erber J (2010) Sucrose acceptance and different forms of associative learning of the honey bee (Apis mellifera L.) in the field and laboratory. Front. Behav. Neurosci. 4:46. doi: 10.3389/ fnbeh.2010.00046

Copyright () 2010 Mujagic, Sarkander, Erber and Erber. This is an open-access article subject to an exclusive license agreement between the authors and the Frontiers Research Foundation, which permits unrestricted use, distribution, and reproduction in any medium, provided the original authors and source are credited. 